\title{
Frequencies and Flutter Speed Estimation for Damaged Aircraft Wing Using Scaled Equivalent Plate Analysis
}

\author{
T. Krishnamurthy ${ }^{*}$ \\ NASA Langley Research Center, Hampton, VA 23681, U.S.A.
}

\begin{abstract}
Equivalent plate analysis is often used to replace the computationally expensive finite element analysis in initial design stages or in conceptual design of aircraft wing structures. The equivalent plate model can also be used to design a wind tunnel model to match the stiffness characteristics of the wing box of a full-scale aircraft wing model while satisfying strength-based requirements An equivalent plate analysis technique is presented to predict the static and dynamic response of an aircraft wing with or without damage. First, a geometric scale factor and a dynamic pressure scale factor are defined to relate the stiffness, load and deformation of the equivalent plate to the aircraft wing. A procedure using an optimization technique is presented to create scaled equivalent plate models from the full scale aircraft wing using geometric and dynamic pressure scale factors. The scaled models are constructed by matching the stiffness of the scaled equivalent plate with the scaled aircraft wing stiffness. It is demonstrated that the scaled equivalent plate model can be used to predict the deformation of the aircraft wing accurately. Once the full equivalent plate geometry is obtained, any other scaled equivalent plate geometry can be obtained using the geometric scale factor. Next, an average frequency scale factor is defined as the average ratio of the frequencies of the aircraft wing to the frequencies of the full-scaled equivalent plate. The average frequency scale factor combined with the geometric scale factor is used to predict the frequency response of the aircraft wing from the scaled equivalent plate analysis. A procedure is outlined to estimate the frequency response and the flutter speed of an aircraft wing from the equivalent plate analysis using the frequency scale factor and geometric scale factor. The equivalent plate analysis is demonstrated using an aircraft wing without damage and another with damage. Both of the problems show that the scaled equivalent plate analysis can be successfully used to predict the frequencies and flutter speed of a typical aircraft wing.
\end{abstract}

\section{Introduction}

Understanding the effects of discrete source damage (e.g., uncontained rotor burst) on the response of aircraft structures is necessary to improve the survivability of future aircraft against damage events. Rapid modeling and analysis methods are among the key requirements for real-time evaluation of damage effects that are needed for integrated vehicle health management. One such analysis method is equivalent plate analysis.

Equivalent plate analysis is often used to replace the computationally expensive finite element analysis in initial design stages or in conceptual design of aircraft wing structures [1]. In equivalent plate modeling, the model characteristics are represented with polynomials, which require only a small fraction of the input data that would be required by a corresponding finite element model. The equivalent plate model can also be used to design a wind tunnel model to match the stiffness characteristics of the wing box of a full-scale aircraft wing model while satisfying strength-based requirements [2]. An equivalent plate analysis procedure based on the Ritz method was proposed at NASA Langley Research Center as early as 1986 [3]. In the Ritz method-based equivalent plate theory, the aircraft wing structure is modeled with several trapezoidal segments. Several modifications and improvements to the initial theory proposed in reference 3 resulted in the development of a structural analysis code ELAPS (Equivalent Laminated Plate Solution) [4, 5]. The ELAPS code solutions predict the displacement, stress, and mode shape calculations within five to ten percent of a comparable finite element solution [6]. However, the Ritz methodbased equivalent plate theory in the ELAPS code is not easily amenable to implement in general-purpose

\footnotetext{
* Aerospace Engineer, Durability, Damage Tolerance and Reliability Branch, Associate Fellow, AIAA
} 
commercial structural finite element analysis codes. Moreover, most of the equivalent plate models including the one implemented in the ELAPS code use theoretical material and section properties, hence it is not always possible to fabricate and test the equivalent plate models. Hence, there is a need to develop equivalent plate models that can be fabricated and tested. Such a research effort is ongoing at NASA Langley Research Center (LaRC). It is also the purpose of this research to establish that scaled equivalent plate models can be used to study the effect of damage on structural behavior.

This is the fourth in a series of papers published [7-9] for the equivalent plate analysis research being performed at NASA Langley Research Center. The equivalent plate analysis research is performed as part of the Integrated Resilient Aircraft Control (IRAC) and the Integrated Vehicle Health Management (IVHM) projects under the NASA Aviation Safety Program. In reference 7, an equivalent plate procedure was developed to provide a computationally efficient means of matching the stiffness and natural frequencies of aircraft wing structures for prescribed loading conditions on simple stiffened-panel geometries. First, the equivalent plate was used to match the stiffness of a stiffened-panel with and without damage. Once the stiffness was matched, the equivalent plate models were then used to predict the frequencies of the panels. Two analytical procedures using the lumped-mass matrix were used to match the first five frequencies of the corresponding detailed model. In both of the procedures, the lumped-mass matrix for the equivalent plate was constructed by multiplying the diagonal terms of the consistent-mass matrix by the proportionality constant. It was found that varying the density alone for the metallic equivalent plate did not provide enough flexibility to match all the frequencies. Hence, composite and sandwich materials for equivalent plate construction were evaluated in reference 8 . Two approaches were presented to match the frequency response of the aircraft wing structures using an equivalent plate model. In the first approach, the equivalent plate was generated by matching only the stiffness of the aircraft wing structure and then the frequencies were matched by adding point masses at discrete locations along the equivalent plate. The second approach generated the equivalent plate by matching both the stiffness and the frequencies of the aircraft wing structure simultaneously. Several candidates for the equivalent plate were considered including composite material, foam core sandwich, and multizone metallic materials. In reference 9 , the equivalent plate procedure developed for full-scale equivalent plate models in references 7 and 8 was extended to construct scaled equivalent plate models. Geometric scale and frequency scale factors were defined to construct an equivalent plate with any desired scale to use in simulation and wind tunnel experiments. It was shown that the stiffness and the displacements were scaled linearly with the geometric scale factor, whereas the load was scaled as the square of the geometric scale factor. The scaled stiffness of the reference aircraft wing was first matched to construct the equivalent plate. Then, the frequency scale factor was defined to scale the aircraft wing frequencies. The scaled aircraft wing frequencies were matched by placing arbitrary point masses along the equivalent plate geometry. The procedures were demonstrated on a typical aircraft wing without damage. Even though the equivalent plate predicted the response of the aircraft wing accurately in reference 9, very heavy masses of up to ten times the equivalent plate's total mass needed to be placed. The purpose of this paper is to extend the work performed in reference 9:

1. To find an alternate procedure to eliminate the heavy masses that need to be added to the plate to match the frequencies.

2. To establish procedures to estimate the flutter speed and flutter frequency of aircraft wings using equivalent plate models.

3. To demonstrate the equivalent plate procedures on an aircraft wing with large damage.

4. To demonstrate that the frequencies and flutter speed of the aircraft wing can be estimated from the frequencies and flutter speed of the scaled equivalent plate models.

First, a geometric scale factor is defined. Next, an optimization procedure to predict the static response of the aircraft wing structures is presented. Third, an average frequency scale factor will be defined to estimate the frequencies of the wing from equivalent plate models. Fourth, the equivalent plate procedure is demonstrated on model of an aircraft wing structure with and without damage. Finally, a brief summary is presented.

\section{Geometric Scale Factor, Dynamic Pressure Scale Factor, and Scaled Stiffness Matching}

It is often necessary to construct a scaled-down equivalent plate model to predict the static response of aircraft wing structures in simulation and in wind tunnel experiments. To simulate the lift, drag, and moment characteristics of an aircraft wing structure using an equivalent plate, it is sufficient to simulate the static load-deflection of the 
wing structure [9]. The stiffness of the equivalent plate and the aircraft wing structure are similar when the nondimensional lift distribution $C_{p}(x, y)$ produces the same nondimensional deflection [10] such that

$\frac{w_{m}}{\sqrt{A_{m}}}=\frac{w_{f}}{\sqrt{A_{f}}}$

where,

$w_{m}-$ is the scaled deflection of the equivalent plate

$w_{f}-$ is the actual deflection of the aircraft wing

$A_{m}-$ is the scaled wing area of the equivalent plate

$A_{f}-$ is the actual wing area of the aircraft wing

The subscripts $m$ and $f$ in Eq. (1) represent the scaled equivalent plate model and the full aircraft wing structure, respectively. At this point, a geometric scale factor $S_{g}$ is selected such that

$$
\begin{aligned}
& S_{g}=\sqrt{\frac{A_{m}}{A_{f}}} \\
& \frac{\left(\Delta A_{m}\right)_{i}}{A_{m}}=\frac{\left(\Delta A_{f}\right)_{i}}{A_{f}}
\end{aligned}
$$

where $\left(\Delta A_{m}\right)_{i}$ and $\left(\Delta A_{f}\right)_{i}$ are the corresponding elemental areas located at a given panel point $i$. The equations (2) and (3) imply that the equivalent plate is uniformly scaled in both the length and the width direction of the aircraft wing geometry.

The aerodynamic load, $\left(P_{i}\right)$, at any elemental area $\Delta A_{i}$ located at a given panel point $i$ is:

$$
P_{i}=q C_{P_{i}} \frac{\Delta A_{i}}{A} A
$$

where $q$ is the dynamic pressure.

This load at point $i$ produces a deflection $w_{j}$ at an arbitrary point $j$. The load-deflection relationship in terms of the stiffness $K_{i j}$ can be written as

$$
P_{i}=K_{i j} w_{j}
$$

The nondimensional deflection in Eq. (1) can be rewritten using Eqs. (4) and (5) as

$$
\frac{w}{\sqrt{A}}=\frac{q C_{p}}{K} \frac{\Delta A}{A} \frac{A}{\sqrt{A}}
$$

In representing Eq. (6), the subscripts $i$ and $j$ are dropped and the equation is valid for both the equivalent plate and full aircraft wing. Neglecting the effect of Reynolds number on $C_{p}$, there will be aerodynamic flow similarity (equal $C_{p}$ ) between the aircraft wing structure and the equivalent plate model, if the models are tested at the same Mach number [10]. Using Eqs. (3) and (6) in Eq. (1), the similarity requirement for the stiffness between the equivalent plate model and the aircraft wing can be written as:

$$
\frac{q_{m}}{q_{f}} \frac{K_{f}}{K_{m}}\left(\frac{A_{m}}{A_{f}}\right)^{\frac{1}{2}}=1
$$

where, 


$$
\begin{aligned}
& q_{m}-\text { is the dynamic pressure of the equivalent plate } \\
& q_{f}-\text { is the dynamic pressure of the aircraft wing } \\
& K_{m}-\text { is the stiffness of the equivalent plate } \\
& K_{f}-\text { is the stiffness of the aircraft wing }
\end{aligned}
$$

For every choice of Mach number and altitude of the airplane, one can select a corresponding Mach number and the dynamic pressure for the equivalent plate model [11]. At this point, the dynamic pressure scale is defined such that

$$
S_{q}=\frac{q_{m}}{q_{f}}
$$

Substituting Eq. (2) and Eq. (8) in Eq. (7), the scaled stiffness for the equivalent plate $K_{m}$ can be expressed in terms of the aircraft wing stiffness $K_{f}$ as

$$
K_{m}=S_{g} S_{q} K_{f}
$$

The relationship between the deformation of the scaled equivalent plate and the deformation of the aircraft wing can be written using Eqs. (1) and (2) as

$$
w_{m}=S_{g} w_{f}
$$

Similarly, the relationship between the applied load $P_{m}$ on the equivalent plate and the applied load on the aircraft wing $P_{f}$ can be written using Eqs. (5) and (9) as

$$
P_{m}=S_{g}^{2} S_{q} P_{f}
$$

Eqs. (9) to (11) completely define the quantities needed for the equivalent plate construction. Once the geometric scale factor $S_{g}$ and dynamic pressure scale factor $S_{q}$ are selected, the load, stiffness, and deformation of the scaled equivalent plate can be determined using Eqs. (9) to (11).

Once the load and displacement of the scaled equivalent plate are determined, the following procedure adopted from references 7 and 8 is used to construct the equivalent plate by finding the optimum thickness distribution of the plate:

1. Define or select the geometric scaling $S_{g}$ and dynamic pressure scaling $S_{q}$.

2. Obtain the displacement field of the aircraft wing by performing a full-scale finite element linear static analysis for the given loading and boundary conditions.

3. Using the geometric scaling, fix the dimensions of the equivalent plate planform.

4. Construct the finite element model of the equivalent plate.

5. If the nodal locations of the equivalent plate model are different from finite element nodes of the full-scale aircraft wing, interpolation should be used to obtain the displacements at the equivalent plate nodal locations. In the present study, the nodes in the equivalent plate are located at the same location as the reference aircraft wing nodes; hence, no interpolation was used.

6. The applied load for the equivalent plate is obtained using $P_{m}=S_{g}^{2} S_{q} P_{f}$.

7. Find the displacement distribution of the equivalent plate by performing linear static analysis.

8. The optimum thickness distribution of the equivalent plate is determined by minimizing the sum of the differences between the displacements of the aircraft wing and the equivalent plate model.

The objective function $\Phi$ for the optimization problem can be stated as

where,

$$
\Phi=\sum_{i=1}^{N}\left(w_{m}^{i}-S_{g} w_{m f}^{i}\right)^{2}
$$

$N \quad$ - is the number of finite element nodes in the equivalent plate model; 
$w_{m}^{i}$ - is the displacement at the $i^{\text {th }}$ node of the equivalent plate; and

$w_{f}^{i}-$ is the interpolated displacement of the aircraft wing at the location of the $i^{t h}$ node of the equivalent plate.

The objective function $\Phi$ in Eq. (12) is minimized in the optimization procedure to obtain the thickness distribution of the equivalent plate subject to the load constraint

$$
P_{m}=S_{g}^{2} S_{q} P_{f}
$$

The optimized thickness distribution of the equivalent plate will produce the reduced stiffness of the scaled equivalent plate as

$$
K_{m}=S_{g} S_{q} K_{f}
$$

Eq. (14) can be modified to obtain the scaled equivalent plate in terms of the full equivalent plate model as

$$
K_{\text {scaled Eq }}=S_{g} S_{q} K_{\text {Full Eq }}
$$

Eq. (15) along with Eq. (1) establishes that the scaled equivalent plate geometry can be obtained from the full equivalent plate geometry by linearly scaling all the dimensions of the full plate with the geometric scale factor.

In the present study, the general purpose finite element code ABAQUS ${ }^{\circledR}[12]^{\dagger}$ is used for the finite element analysis and the optimization code $\mathrm{DOT}^{\circledR}[13]$ is used in the optimization procedure.

\section{Frequency and Flutter Scaling between Wing and Full-Scale Equivalent Plate}

The natural frequencies of two elastic structures having the same stiffnesses but with different masses will differ by a constant ratio. Since the stiffness of the equivalent plate and the wing are matched only in an approximate sense, the ratio of natural frequencies of the equivalent plate and the wing will not be a constant in general. Hence, an average frequency scale factor $\left(S_{\omega}\right)$ is defined as the average ratios of the frequencies of the aircraft wing to the frequencies of the full-scale equivalent plate

$$
\begin{aligned}
& S_{\omega}=\operatorname{Ave}\left(\frac{\omega_{\text {wing }_{i}}}{\omega_{F u l l E q_{i}}}\right) \\
& \text { where, } \\
& \omega_{\text {wing }} \text { is the natural frequency of the aircraft wing } \\
& \omega_{\text {Full Eq }} \text { is the natural frequency of the full-scale equivalent plate }
\end{aligned}
$$

The predicted natural frequency of the wing is obtained by scaling the full-scale equivalent plate frequencies by the average frequency scale factor

$$
\omega_{\text {wing }} \approx S_{\omega} \omega_{F u l l E q}
$$

Flutter analysis was performed using NASTRAN ${ }^{\circledR}[14]$ to determine the flutter frequency and speed of the wing and equivalent plate. Similar to the scaling of the natural frequency of the wing to the full-scale equivalent plate, the flutter frequency $(\Omega)$ also differs by $S_{\omega}$.

$$
\Omega_{\text {wing }} \approx S_{\omega} \Omega_{\text {Full Eq }}
$$

\footnotetext{
${ }^{\dagger}$ Trade names and trademarks are used in this report for identification only. Their usage does not constitute an official endorsement, either expressed or implied, by the National Aeronautics and Space Administration.
} 
The flutter speed of the wing is approximately equal to the flutter speed of the full-scale equivalent plate.

$$
\begin{aligned}
& \mathrm{m}_{\text {wing }} \approx \mathrm{m}_{\text {Full Eq }} \\
& \text { where, }
\end{aligned}
$$

$m$ is the ratio between the flutter speed and the aircraft operating speed.

The approximation in the Eqs. (17) to (19) are due to the possible error in matching the stiffness distribution and mass distribution of the equivalent plate with respect to the aircraft wing.

\section{Frequency and Flutter Scaling between Full-Scale Equivalent Plate and Scaled Equivalent Plate}

The scaled equivalent plate geometry is obtained by scaling the full-scale equivalent plate by the geometric scale factor. Thus, the frequency of the full-scale equivalent plate and the scaled equivalent plate $\left(\omega_{\text {Scaled Eq }}\right)$ only differs by $S_{g}$ (using Eqs. 3 and 9),

$$
\omega_{\text {Full Eq }}=S_{g} \omega_{\text {Scaled Eq }}
$$

Similarly, scaling of the flutter frequency of the full-scale equivalent plate to the scaled equivalent plate $\left(\Omega_{\text {Scaled Eq }}\right)$ differs by $S_{g}$.

$$
\Omega_{\text {Full Eq }}=S_{g} \Omega_{\text {Scaled Eq }}
$$

The flutter speed of the full-scale equivalent plate is approximately equal to the scaled equivalent plate.

$$
\mathrm{m}_{\text {Full Eq }} \approx \mathrm{m}_{\text {Scaled Eq }}
$$

\section{Frequency and Flutter Speed of an Aircraft Wing}

In this paper, the static and dynamic response of an aircraft wing is obtained from the analysis of a scaled equivalent plate. The natural frequencies of the aircraft wing can be obtained from the scaled equivalent plate frequencies using Eqs. (17) and (20) as

$$
\omega_{\text {wing }} \approx S_{g} S_{\omega} \omega_{\text {Scaled Eq }}
$$

Similarly, the flutter frequency can be obtained from the scaled equivalent plate frequency using Eqs. (17) and (20) as

$$
\Omega_{\text {wing }} \approx S_{g} S_{\omega} \Omega_{\text {Scaled Eq }}
$$

The flutter speed can be obtained from the scaled equivalent plate frequency using Eqs. (18) and (21) as

$$
\mathrm{m}_{\text {wing }} \approx \mathrm{m}_{\text {Scaled Eq }}
$$

The approximations in Eqs. (23) to (25) will be validated through numerical examples in this paper.

\section{Typical Aircraft Wing Structure with No Damage}

The methodology employing geometric and frequency scale factors to predict the static and dynamic response is demonstrated on a typical aircraft wing structure. A detailed finite element model of the typical aircraft wing with no damage used in the demonstration is shown in Figure 1. The model has 2 spars and 26 ribs. The overall 
dimensions of the wing are also shown in Figure 1. An equivalent plate model was created by extracting the elements of the upper skin and zeroing out the vertical coordinates of all nodes (not shown here).

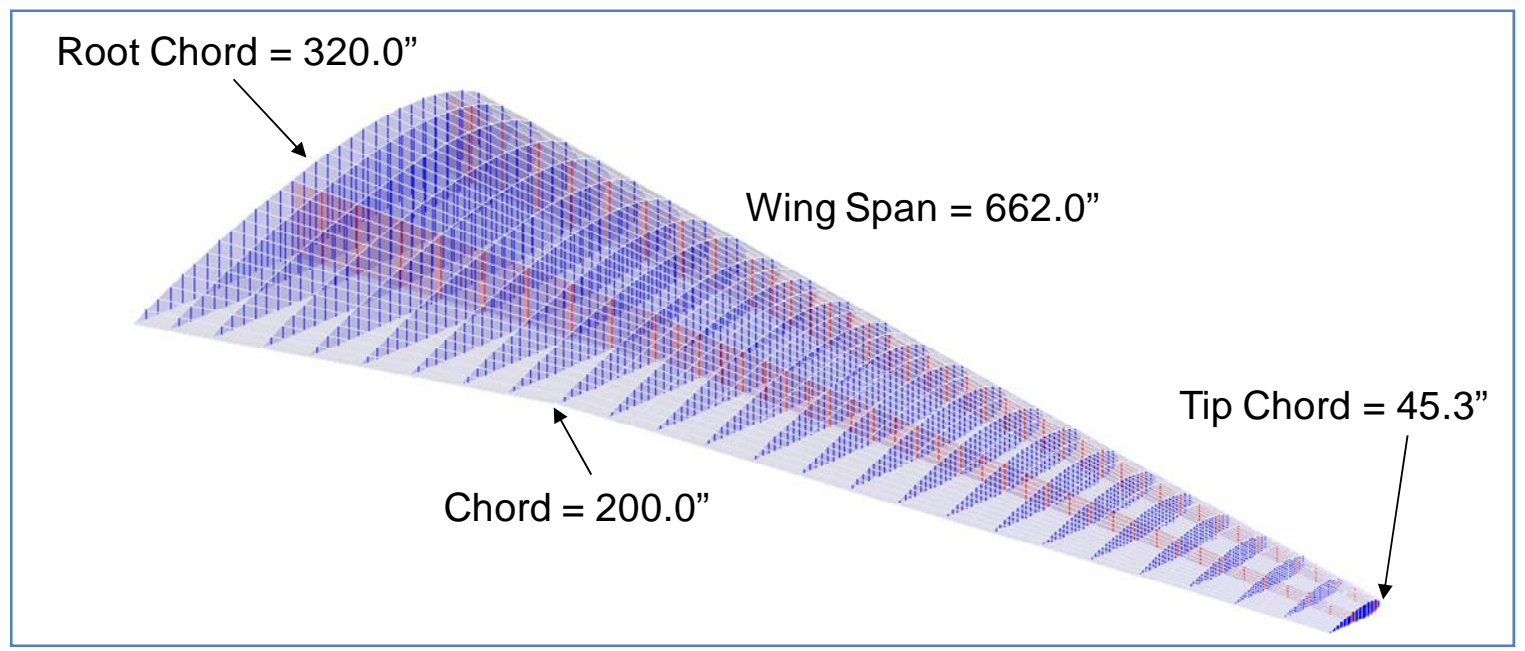

Figure 1. Typical Aircraft Wing with No Damage: Finite Element Model

First, equivalent plate geometries were generated by matching the stiffness of the aircraft wing structure for geometric scale factors 1.0 and 0.2 by using procedures developed in Section II, while keeping the dynamic pressure scale factor equal to 1.0. Figure 2 shows the deformations of the wing and equivalent plates. The deformation pattern from the equivalent plate (scaled deformation for the fifth-scale equivalent plate) is in very good agreement with the deformation pattern from the aircraft wing structure.

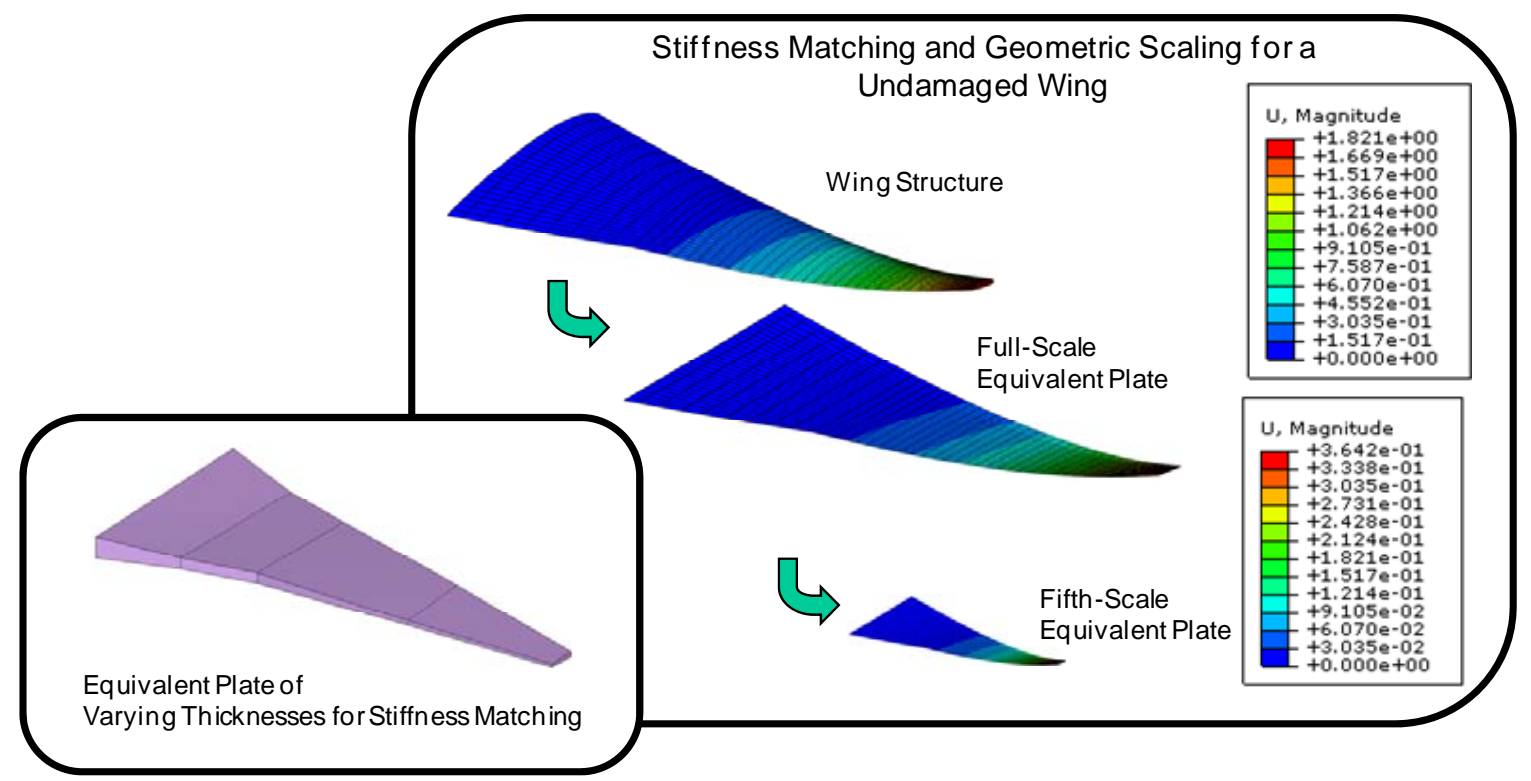

Figure 2. Aircraft Wing Structure: Deformation Comparison for Geometric-Scale Factors 1.0 and 0.2

Once the full scale equivalent plate geometry is obtained, the average frequency scale factor $\left(S_{\omega}\right)$ in Eq. (16) is obtained by performing frequency response (Eigenvalue) calculation. The natural frequencies obtained from the wing and the full scale equivalent plate are given in Table 1. If the stiffness and the mass distribution of the full scale equivalent plate is exactly matched with that of the aircraft wing, the ratios of the natural frequencies of the wing to the equivalent plate will be constant for all the frequencies. The ratios of the natural frequencies are not constant, since the equivalent plate stiffness is matched only approximately. Hence, an average frequency scale factor is obtained as 2.82 . 
Table 1. Frequency Response of the Aircraft Wing and Full Scale Equivalent Plate

\begin{tabular}{|c|c|c|c|}
\cline { 2 - 4 } \multicolumn{1}{c|}{} & $\begin{array}{c}\text { Natural Frequency for } \\
\text { the Full Scale Wing } \\
\left(\boldsymbol{\omega}_{\text {wing }}\right)\end{array}$ & $\begin{array}{c}\text { Natural Frequency of } \\
\text { the Full Scale Eq Plate } \\
\left(\boldsymbol{\omega}_{\text {Eq Plate }}\right)\end{array}$ & $\frac{\boldsymbol{\omega}_{\text {wing }}}{\boldsymbol{\omega}_{\text {Eq Plate }}}$ \\
\hline Mode 1 & $6.37 \mathrm{~Hz}$ & $2.13 \mathrm{~Hz}$ & 2.99 \\
\hline Mode 2 & $16.02 \mathrm{~Hz}$ & $5.45 \mathrm{~Hz}$ & 2.94 \\
\hline Mode 3 & $31.7 \mathrm{~Hz}$ & $10.74 \mathrm{~Hz}$ & 2.95 \\
\hline Mode 4 & $42.12 \mathrm{~Hz}$ & $15.59 \mathrm{~Hz}$ & 2.70 \\
\hline Mode 5 & $47.98 \mathrm{~Hz}$ & $18.77 \mathrm{~Hz}$ & 2.56 \\
\hline \multicolumn{2}{|c|}{ Average frequency scale factor $=S_{\omega}=\frac{\frac{\omega_{\text {wing }}}{\omega_{E q \text { Plate }}}}{N}$} & $; \quad$ with $N=5 ;$ & $S_{\omega}=2.82$ \\
\hline
\end{tabular}

Next, the scaled equivalent plate model was used to obtain the natural frequency of the aircraft wing using Eq. (17). The equivalent plate frequencies along with the frequency scale factor $S_{\omega}=2.82$, are used to predict the frequency response of the aircraft wing. The predicted frequencies of the aircraft wing are compared with the frequencies obtained from the aircraft wing analysis in Figure 3 and found to be within $10 \%$. Hence, it can be concluded that the frequency response of the equivalent plate can be used to predict the frequency response of the aircraft wing with reasonable accuracy.

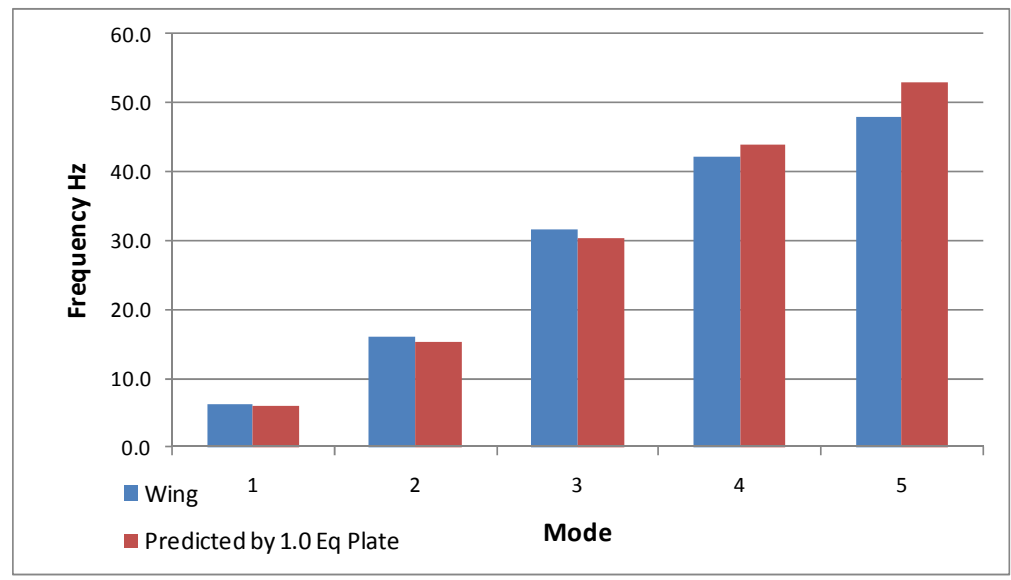

Figure 3. Natural Frequency of Aircraft Wing vs. Frequency Predicted by Equivalent Plate 
Lastly, the flutter frequency and speed of the aircraft wing and scaled equivalent plates were determined. The flutter analysis performed is for an aircraft operating speed of Mach 0.8 at an elevation of 20,000 ft. The scaled equivalent plate flutter results were used to predict the flutter frequency and speed of the aircraft wing according to procedures developed in Sections III and IV. The calculated average frequency scale factor of 2.82 (Table 1) is used in the calculation. The calculated flutter speed of the aircraft wing is compared with the predicted flutter speed of the aircraft wing from the equivalent plate analysis in Table 2. From Table 2, it can be stated that the predicted aircraft wing flutter speed 5.1 from the equivalent plate analysis is within four percent from the flutter speed of 5.3 obtained from the full aircraft wing analysis.

Table 2. Flutter Frequency and Speed Prediction from Scaled-Equivalent Plate

\begin{tabular}{|c|c|c|c|c|}
\hline & & $\begin{array}{c}\text { Natural } \\
\text { Frequency }(\omega)\end{array}$ & $\begin{array}{c}\text { Flutter } \\
\text { Frequency }(\omega)\end{array}$ & $\frac{\text { Flutter Speed }}{\text { AircraftSpeed }}(\mathrm{m})$ \\
\hline \multicolumn{2}{|c|}{ Fifth-Scale Eq Plate } & $10.67 \mathrm{~Hz}$ & $41.25 \mathrm{~Hz}$ & 5.1 \\
\hline \multicolumn{2}{|c|}{ Full-Scale Eq Plate } & $2.13 \mathrm{~Hz}$ & $8.24 \mathrm{~Hz}$ & 5.1 \\
\hline \multirow{2}{*}{$\begin{array}{l}\text { Wing } \\
\text { Structure }\end{array}$} & $\begin{array}{l}\text { Eq Plate } \\
\text { Analysis }\end{array}$ & $6.01 \mathrm{~Hz}$ & $23.24 \mathrm{~Hz}$ & 5.1 \\
\hline & $\begin{array}{c}\text { Wing } \\
\text { Analysis }\end{array}$ & $6.37 \mathrm{~Hz}$ & $22.25 \mathrm{~Hz}$ & 5.3 \\
\hline
\end{tabular}

\section{Typical Aircraft Wing Structure with Damage}

Next, the equivalent plate analysis will be demonstrated using a model of an aircraft wing with damage. The detailed finite element model of the aircraft wing with damage used in the demonstration is shown in Figure 4. This model was created from the full scale undamaged aircraft wing shown in Figure 1 by introducing a circular hole in the model to simulate the damage. The internal structure and overall dimensions of the model are the same as the wing with no damage in Figure 1. The damage, as represented by a circular hole, severs the rear spar and three ribs internally. An equivalent plate model was created by extracting the elements of the upper skin and zeroing out the vertical coordinates of all nodes (not shown here).

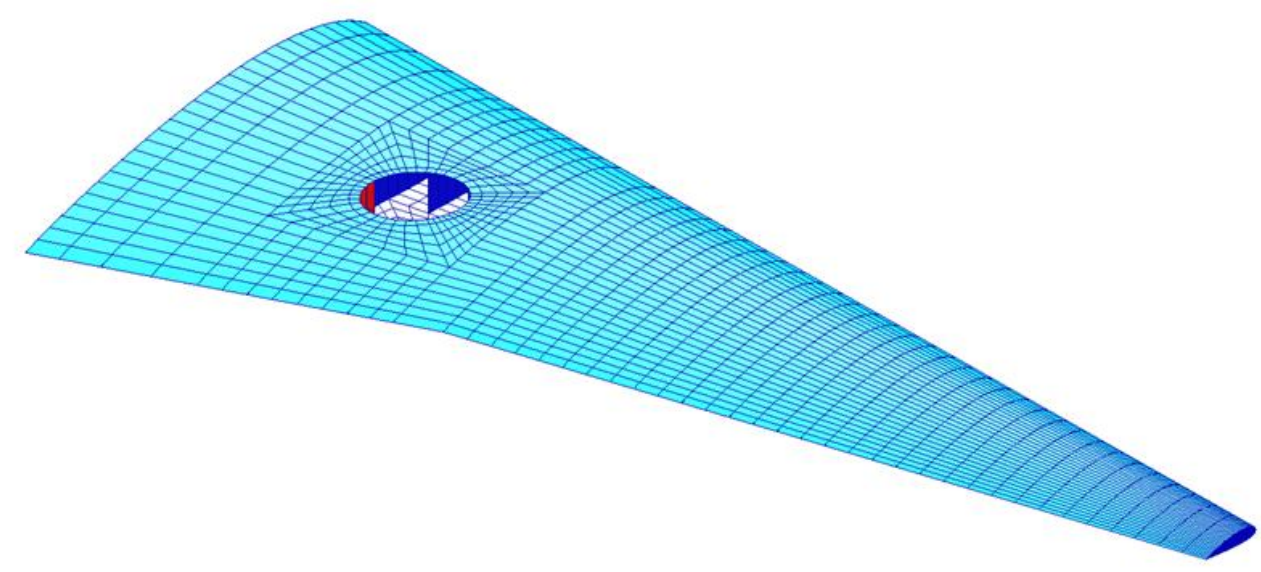

Figure 4. Typical Aircraft Wing with Damage: Finite Element Model 
Again, equivalent plate geometries were generated by matching the stiffness of the aircraft wing structure for geometric scale factors 1.0 and 0.2 by using procedures developed in Section II, while keeping the dynamic pressure scale factor equal to 1.0. Figure 5 shows the deformations of the wing and equivalent plates. For the damaged wing also, the deformation pattern from the equivalent plate (scaled deformation for the fifth-scale equivalent plate) is in very good agreement with the deformation pattern from the aircraft wing structure.

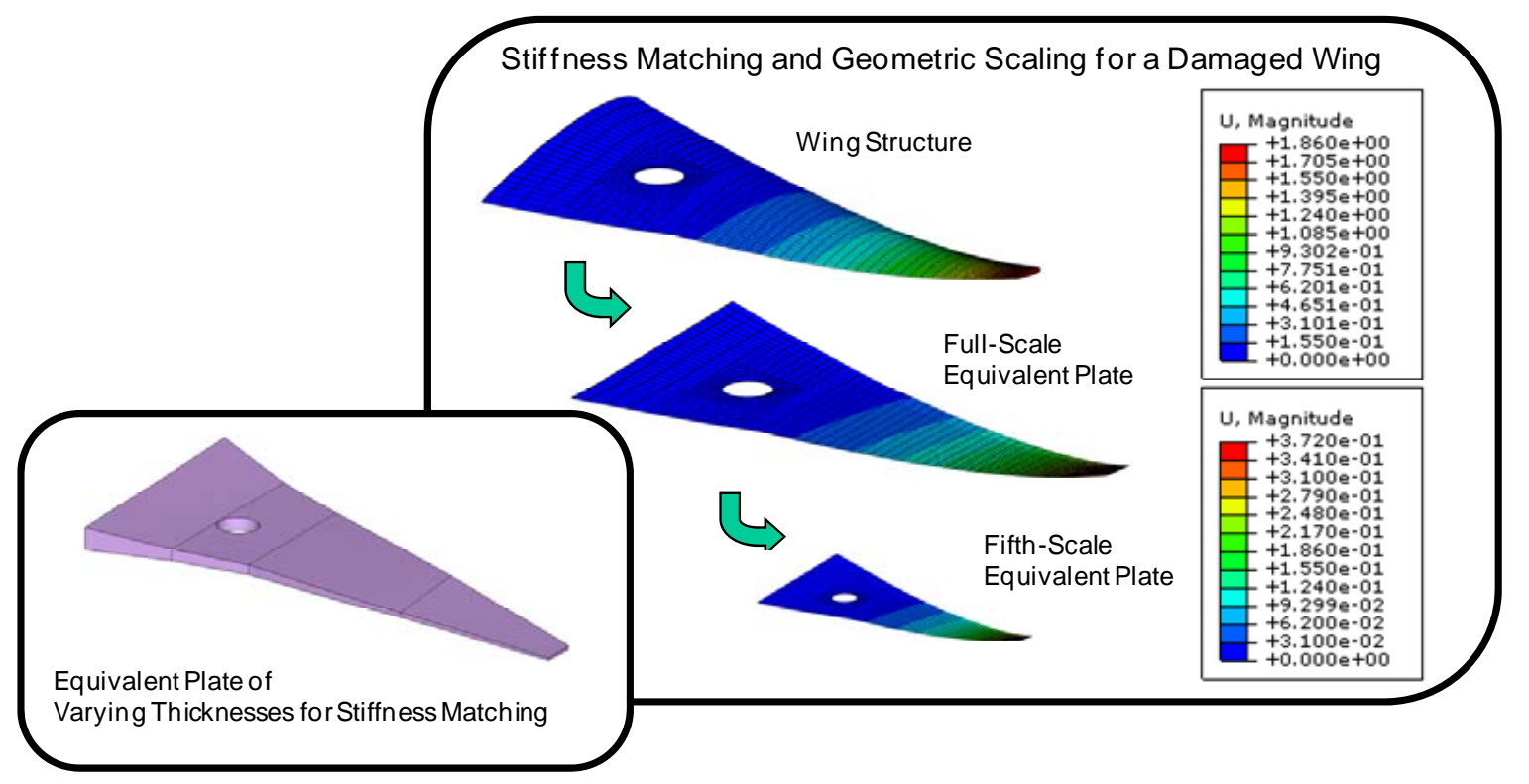

Figure 5. Aircraft Wing Structure: Deformation Comparison for Geometric-Scale Factors 1.0 and 0.2

As before, once the full scale equivalent plate geometry is obtained, the average frequency scale factor $\left(S_{\omega}\right)$ in Eq. (16) is obtained by performing a frequency response (Eigenvalue) calculation. The natural frequencies obtained from the wing and the full scale equivalent plate are given in Table 3. The calculated average frequency scale factor is also shown in Table 3. As before for the undamaged wing example, the ratios between the natural frequencies of the wing and the equivalent plate are not constant, since the equivalent plate stiffness is matched only approximately in the procedure.

The scaled equivalent plate model was used to predict the natural frequency of the aircraft wing structure. The predicted frequencies of the aircraft wing are compared to the frequencies obtained from the aircraft wing analysis in Figure 6 and found to be within 8\%. It is clearly demonstrated that the equivalent plate analysis can be used to predict the frequency response of the aircraft wing with or without damage.

The flutter frequency and speed of the aircraft wing and scaled equivalent plates were determined using NASTRAN. The flutter analysis performed is for an aircraft operating speed of Mach 0.8 at an elevation of 20,000 $\mathrm{ft}$. The scaled equivalent plate flutter results were scaled according to procedures developed in Sections III and IV to predict the flutter frequency and speed of the aircraft wing, which are shown in Table 4 . Here again, the predicted aircraft wing flutter speed 5.1 from the equivalent plate analysis is within four percent from the flutter speed of 4.9 obtained from the full aircraft wing analysis. The results demonstrate that the equivalent plate analysis can be used to predict the flutter speed of the aircraft wing with reasonable accuracy. 
Table 3. Frequency Response of the Aircraft Wing and Full Scale Equivalent Plate

\begin{tabular}{|c|c|c|c|}
\cline { 2 - 4 } \multicolumn{1}{c|}{} & $\begin{array}{c}\text { Natural Frequency for } \\
\text { the Full Scale Wing } \\
\left(\boldsymbol{\omega}_{\text {wing }}\right)\end{array}$ & $\begin{array}{c}\text { Natural Frequency of } \\
\text { the Full Scale Eq Plate } \\
\left(\boldsymbol{\omega}_{\text {Eq Plate }}\right)\end{array}$ & $\frac{\boldsymbol{\omega}_{\text {wing }}}{\boldsymbol{\omega}_{\text {Eq Plate }}}$ \\
\hline Mode 1 & $6.14 \mathrm{~Hz}$ & $2.11 \mathrm{~Hz}$ & 2.91 \\
\hline Mode 2 & $14.99 \mathrm{~Hz}$ & $5.55 \mathrm{~Hz}$ & 2.70 \\
\hline Mode 3 & $28.72 \mathrm{~Hz}$ & $10.87 \mathrm{~Hz}$ & 2.64 \\
\hline Mode 4 & $40.56 \mathrm{~Hz}$ & $15.36 \mathrm{~Hz}$ & 2.64 \\
\hline Mode 5 & $46.86 \mathrm{~Hz}$ & $18.87 \mathrm{~Hz}$ & 2.48 \\
\hline Average frequency scale factor $=S_{\omega}=\frac{\sum_{\omega_{\text {Eq Plate }}}}{N}$ & $;$ & with $N=5 ;$ & $S_{\omega}=2.67$ \\
\hline
\end{tabular}

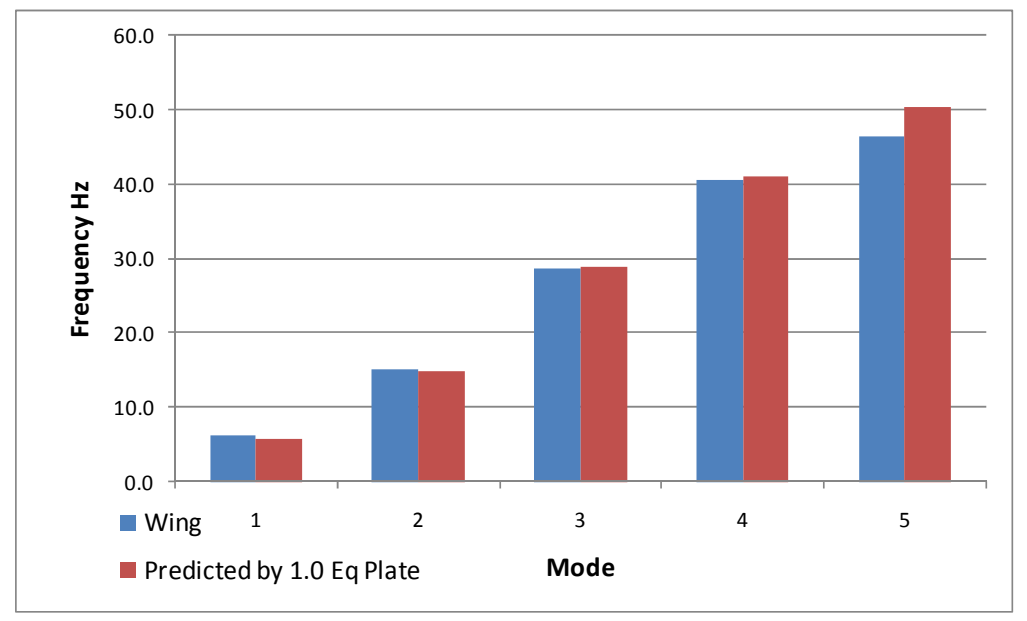

Figure 6. Natural Frequency of Aircraft Wing vs. Frequency Predicted by Equivalent Plate 
Table 4. Flutter Frequency and Speed Prediction from Scaled-Equivalent Plate

\begin{tabular}{|c|c|c|c|c|}
\hline & & $\begin{array}{c}\text { Natural } \\
\text { Frequency }(\omega)\end{array}$ & $\begin{array}{c}\text { Flutter } \\
\text { Frequency }(\boldsymbol{\omega})\end{array}$ & $\begin{array}{l}\text { Flutte Speed } \\
\text { Aircraft Speed }\end{array}$ \\
\hline \multicolumn{2}{|c|}{ Fifth-Scale Eq Plate } & $10.57 \mathrm{~Hz}$ & $40.33 \mathrm{~Hz}$ & 5.1 \\
\hline \multicolumn{2}{|c|}{ Full-Scale Eq Plate } & $2.11 \mathrm{~Hz}$ & $8.06 \mathrm{~Hz}$ & 5.1 \\
\hline \multirow{2}{*}{$\begin{array}{l}\text { Wing } \\
\text { Structure }\end{array}$} & $\begin{array}{l}\text { Eq Plate } \\
\text { Analysis }\end{array}$ & $5.64 \mathrm{~Hz}$ & $21.51 \mathrm{~Hz}$ & 5.1 \\
\hline & $\begin{array}{l}\text { Wing } \\
\text { Analysis }\end{array}$ & $6.14 \mathrm{~Hz}$ & $20.04 \mathrm{~Hz}$ & 4.9 \\
\hline
\end{tabular}

\section{Summary}

An equivalent plate analysis technique is presented to predict the static and dynamic response of an aircraft wing with or without damage. First, a geometric scale factor and a dynamic pressure scale factor are defined to relate the stiffness, load and deformation of the equivalent plate to the aircraft wing. A procedure using an optimization technique is presented to create scaled equivalent plate models from the full scale aircraft wing using geometric and dynamic pressure scale factors. The scaled equivalent plate models are constructed by matching the stiffness of the scaled equivalent plate with the scaled aircraft wing stiffness. It is demonstrated that the scaled equivalent plate model can be used to predict deformation of the aircraft wing accurately. Once the full scale equivalent plate geometry is obtained, any other scaled equivalent plate geometry can be obtained using the geometric scale factor.

Next, an average frequency scale factor is defined as the average ratios of the frequencies of the aircraft wing to the frequencies of the full-scale equivalent plate. The average frequency scale factor combined with the geometric scale factor is used to predict the frequency response of the aircraft wing from the scaled equivalent plate analysis.

A procedure is outlined to estimate the frequency response and the flutter speed of an aircraft wing from the equivalent plate analysis using the frequency scale factor and geometric scale factor.

The equivalent plate analysis is demonstrated using an aircraft wing without damage and another with damage. Both of the problems showed that the scaled equivalent plate analysis can be successfully used to predict the static and dynamic response of a typical aircraft wing within 5 percent. The frequency response and flutter speed of an aircraft wing can be estimated from a scaled equivalent plate model analysis with reasonable accuracy. 


\section{References}

1. Giles, Gary L., "Equivalent Plate Modeling for Conceptual Design of Aircraft Wing Structures,” AIAA1995-3945, Presented at $1^{\text {st }}$ AIAA Aircraft Engineering, Technology and Operations Congress, Sept. 19-21, 1995, Los Angeles, CA.

2. Mason, B., Stroud, W., Krishnamurthy, T., Spain, C., and Naser A., "Probabilistic Design of a Wind Tunnel Model to Match the Response of a Full-Scale Aircraft,” AIAA-2005-2185, Presented at 46th AIAA/ASME/ASCE/AHS/ASC Structures, Structural Dynamics and Materials Conference 13th AIAA/ASME/AHS Adaptive Structures Conference, Austin, Texas, Apr. 18-21, 2005.

3. Giles, Gary L., "Equivalent Plate Analysis of Aircraft Wing Box Structures with General Planform Geometry,” Journal of Aircraft, Vol. 23. No. 11, pp. 858-864, 1986.

4. Giles, Gary L., "Further Generalization of Equivalent Plate Representation for Aircraft Structural Analysis,” Journal of Aircraft, Vol. 26. No. 1, pp. 67-74, 1989.

5. Stone, Steven C., Henderson, Joseph L., Nazari, Mark M., Boyd, William N., Becker, Bradley T., Bhatia, Kumar G., Giles, Gary L., and Wrenn, Gregory A., "Evaluation of Equivalent Plate Solution (ELAPS) in HSST Sizing,” AIAA-2000-1452, Presented at 41st AIAA/ASME/ASCE/AHS/ASC Structures, Structural Dynamics, and Materials Conference and Exhibit, 41st, Atlanta, GA, Apr. 3-6, 2000.

6. Mavris, Dimitri N., and Hayden, William T., "Probabilistic Analysis of an HSCT Modeled with an Equivalent Laminated Plate Wing,” AIAA-1997-5571, Presented at AIAA and SAE, 1997 World Aviation Congress, Anaheim, CA, Oct. 13-16, 1997.

7. Krishnamurthy,T., and Mason, Brian H., "Equivalent Plate Analysis of Aircraft Wing With Discrete Source Damage,” AIAA-2006-2218, Presented at 47th AIAA/ASME/ASCE /AHS/ASC Structures, Structural Dynamics and Materials Conference 13th AIAA/ASME /AHS Adaptive Structures Conference, Newport, Rhode Island, May 1-4, 2006.

8. Krishnamurthy,T., and Eldred, Lloyd B., "Frequency Response of an Aircraft Wing with Discrete Source Damage Using Equivalent Plate Analysis, ” AIAA-2007-2144, Presented at 48th AIAA/ASME/ASCE/AHS/ASC Structures, Structural Dynamics, and Materials Conference, Honolulu, Hawaii, Apr. 23-26, 2007.

9. Krishnamurthy,T., and Tsai, Frank J., "Static and Dynamic Structural Response of an Aircraft Wing with Damage Using Equivalent Plate Analysis, ” AIAA-2008-1967, Presented at 49th AIAA/ASME/ASCE/AHS/ASC Structures, Structural Dynamics, and Materials Conference, Schaumburg, IL, Apr. 07-10, 2008.

10. Roskam, J., Holgate, T., and Shimizu, G, "Elastic Wind-Tunnel Models for Predicting Longitudinal Stability Derivatives of Elastic Airplanes,” Journal of Aircraft, Vol. 5, No. 6, pp. 543-550, 1968.

11. Heeg J., Spain, C. V., and Rivera, J. A., "Wind Tunnel to Atmospheric Mapping for Static Aeroelastic Scaling,” AIAA-2004-2044, Presented at 45th AIAA/ASME/ASCE/AHS/ASC Structures, Structural Dynamics, and Materials Conference, Palm Springs, California, Apr. 19-22, 2004.

12. Anonymous, Getting Started with ABAQUS ${ }^{\circledR}$, Version 6.5, ABAQUS, Inc., Providence, RI 02909.

13. Anonymous, $D O T^{\circledR}$, Design Optimization Tools, User's Manual, Version 5.0, Vanderplaats Research \& Development, Inc., Colorado Springs, CO 80906.

14. Rodden, W. P. and Johnson, E. H., MSC/ NASTRAN ${ }^{\circledR}$ Aeroelastic Analysis, User's Guide, Version 68, The MacNeal-Schwendler Corporation, Los Angeles, CA 90041. 